\title{
Thrombosis of the deep dorsal penile vein and corpora cavernosa of the penis as the first symptoms of colon cancer. Case report and literature review
}

\author{
Marek Roslan 1,2, Maciej Przudzik, 2, Michał Borowik, 2, Henryk Wronka3, \\ Zygmunt Kozielec $^{4,5}$, Maciej Michalak ${ }^{6,7}$, Radosław Grabysa ${ }^{3,8}$ \\ 'Department of Urology, Faculty of Medicine, University of Warmia and Mazury, Olsztyn, Poland \\ 2 Department of Urology, Warmian-Masurian Cancer Center of the Ministry of the interior and \\ Administration's Hospital, Olsztyn, Poland \\ ${ }^{3}$ Department of Internal Medicine and Gastroenterology, Warmian-Masurian Cancer Center of the \\ Ministry of the Interior and Administration's Hospital, Olsztyn, Poland \\ ${ }^{4}$ Department of Pathomorphology, Warmian-Masurian Cancer Center of the Ministry of the Interior \\ and Administration's Hospital, Olsztyn, Poland \\ ${ }^{5}$ Department of Pathomorphology, Faculty of Medical Sciences, University of Warmia and Mazury, \\ Olsztyn, Poland \\ ${ }^{6}$ Department of Radiology, Warmian-Masurian Cancer Center of the Ministry of the Interior and \\ Administration's Hospital, Olsztyn, Poland \\ 'Department of Radiology, Faculty of Medical Sciences, University of Warmia and Mazury, Olsztyn, \\ Poland \\ ${ }^{8}$ Department of Pulmonology, Faculty of Medical Sciences, University of Warmia and Mazury, \\ Olsztyn, Poland
}

Correspondence: Marek Roslan Department of Urology, Faculty of Medicine, University of Warmia and Mazury 10-719 Olsztyn, Poland,

Oczapowskiego 2 e-mail:mroslan@gumed.edu.pl phone: +48 895234913

fax: +48895234456

Received:

2.10.2020

Accepted:

10.11.2020

DOI: 10.24292/01.OR.320101120 Copyright $\odot$ Medical Education. All rights reserved.

\section{ABSTRACT}

The case describes an 86-year-old man with thrombosis of the deep dorsal vein and corpora cavernosa of the penis who developed symptoms 4 months before hospitalisation. An accurate imaging diagnostics supplemented with a biopsy of the corpus cavernosum confirmed the initial diagnosis. Moreover, an adenocarcinoma of the hepatic flexion of the colon with numerous metastatic lesions, including those to the crus of penis were found. Diagnostic and therapeutic difficulties were described and the results obtained were discussed in the context of available literature.

Key words: deep dorsal penile vein thrombosis, corpus cavernosus thrombosis, metastatic colorectal cancer 


\section{INTRODUCTION}

The deep dorsal vein of the penis lies centrally in the dorsal part of the penis between the tunica albuginea of the corpora cavernosa and the deep penile fascia (Buck's fascia). The vein transports blood from the glans and corpora cavernosa of the penis to the deep dorsal vein complex (Santorini plexus). Cutting off of the blood outflow in the vein leads to swelling and partial, or complete, penile erection [1]. Symptoms of the penile deep dorsal vein thrombosis (PDDVT) and accompanying corpora cavernosa thrombosis are diagnosed extremely rarely and the aetiology of the disease is not fully understood. The most common symptom observed is moderately painful and relatively hard swelling of the entire, or part of the penis.

Spontaneous occurrences of PDDVT have also been described [1-3]. Differentiation should also take into consideration the thrombosis of the superficial dorsal vein of the penis (Mondor's disease), which is far more prevalent and has a different clinical picture, and pathological penile erection (priapism) [4].

\section{CASE REPORT}

In April 2020, an 86-year-old male patient was admitted to the hospital due to non-painful, hard swelling of the penis and progressing weakening of the urine stream that he had been experiencing for 4 months. The patient claimed there had been no injury and was unable to describe any other tangible reasons of the condition. The patient did not present any other symptoms in the urinary or digestive tract.

During physical examination the penis was found to be swollen in its entire length, non-painful during palpation, without elevation and stiffness typical to erection during intercourse or in priapism (fig. 1). The foreskin was of correct length, however it could not be shifted beyond the groove of the glans. The skin of the penis and scrotum was healthy. Rectal examination showed a smooth, flexible and symmetric prostate with clear boundaries that was enlarged to around $45 \mathrm{~mm}$. The patient presented symmetric, pasty swelling of the lower limbs and suffered from dyspnoea at rest.
FIGURE 1.

The swelling of the penis in corpora cavernosa and deep dorsal penile vein thrombosis.

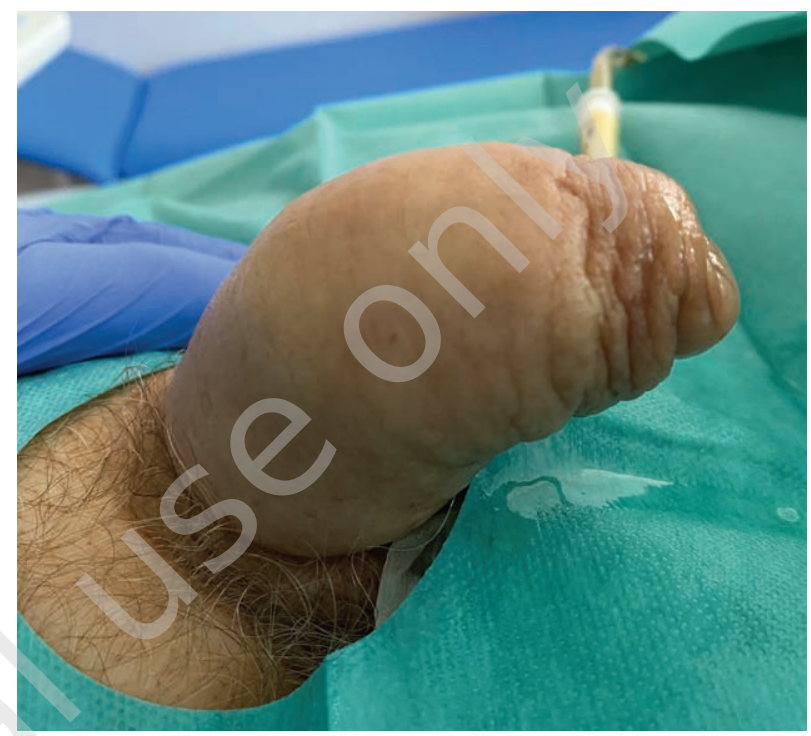

The ultrasound examination of the urinary tract showed normal kidneys, bladder with thick, smooth walls and symmetric prostate of $30 \mathrm{ml}$ capacity. About $150 \mathrm{ml}$ of residual urine was found. Laboratory tests showed evidence of significant anemization $(\mathrm{Hb} 8.8 \mathrm{~g} / \mathrm{dl})$ and an increased concentration of D-dimers (3350).

The patient was catheterised with a $12 \mathrm{Ch}$ Foley catheter - a slight resistance was encountered in the bulbar part of the urethra.

The patient was given a transfusion of 2 units of concentrated red blood cells. After an internal medicine and cardiology consultation and suspecting venous thrombosis of the lower limbs, the patient was given anti-oedema therapy (furosemide) and anticoagulant therapy (low molecular weight heparin).

Doppler examination did not confirm an impaired venous flow in the lower limbs; however, it did detect the lack of flow in the deep dorsal vein of the penis and in the corpora cavernosa of the penis (fig. 2). Computerised tomography of the abdominal cavity and the pelvis detected a tumour of the hepatic flexion of the colon with multiple metastatic lesions in the liver and lungs (fig. 3). Due to ambiguous clinical picture of the changes observed, the patient was qualified for a core-needle biopsy (Tru-Cut needle), which confirmed thrombosis of the corpora cavernosa of the penis. Adenocarcinoma tissue was found in one of the samples collected during biopsy (fig. 4). 
FIGURE 2.

USG colour Doppler examination detecting the lack of flow in deep dorsal penile vein and corpora cavernosa.

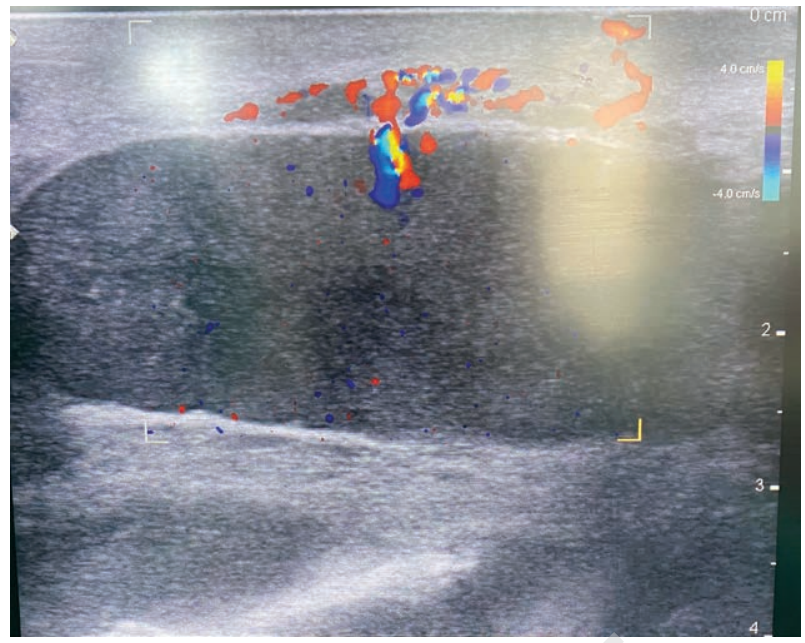

\section{FIGURE 3.}

Computerised tomography of the abdomen and pelvis. Visible tumour of the hepatic flexion of the colon and the numerous metastases in the liver.



FIGURE 4.

Core needle biopsy. The image of adenocarcinoma metastasis in the corpora cavernosa of the penis.

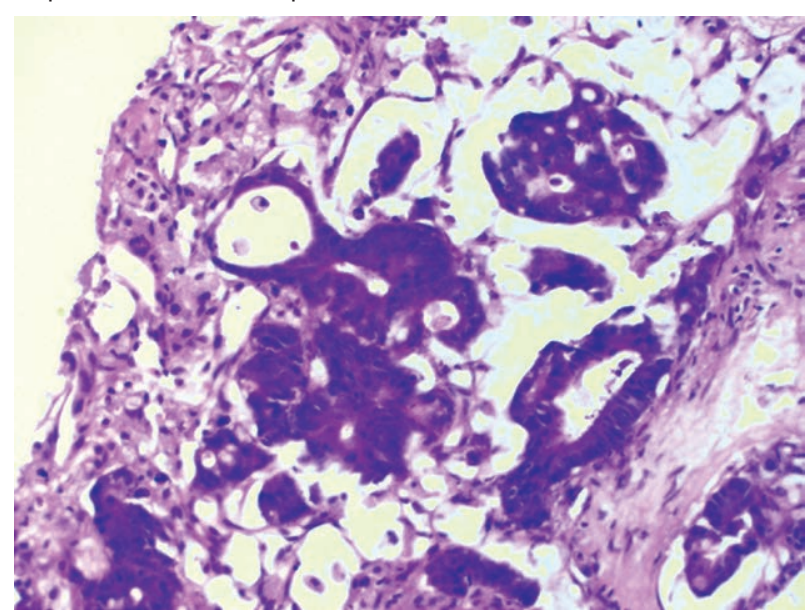

Magnetic resonance imaging (MRI) of the pelvis detected a $3 \mathrm{~cm}$ metastatic lesion in the base of the penis and in both corpora cavernosa (fig. 5).

\section{FIGURE 5.}

MRI imaging of the pelvis and penis. Visible thrombotic changes in the corpora cavernosa (arrows) and adenocarcinoma metastasis (triangles).

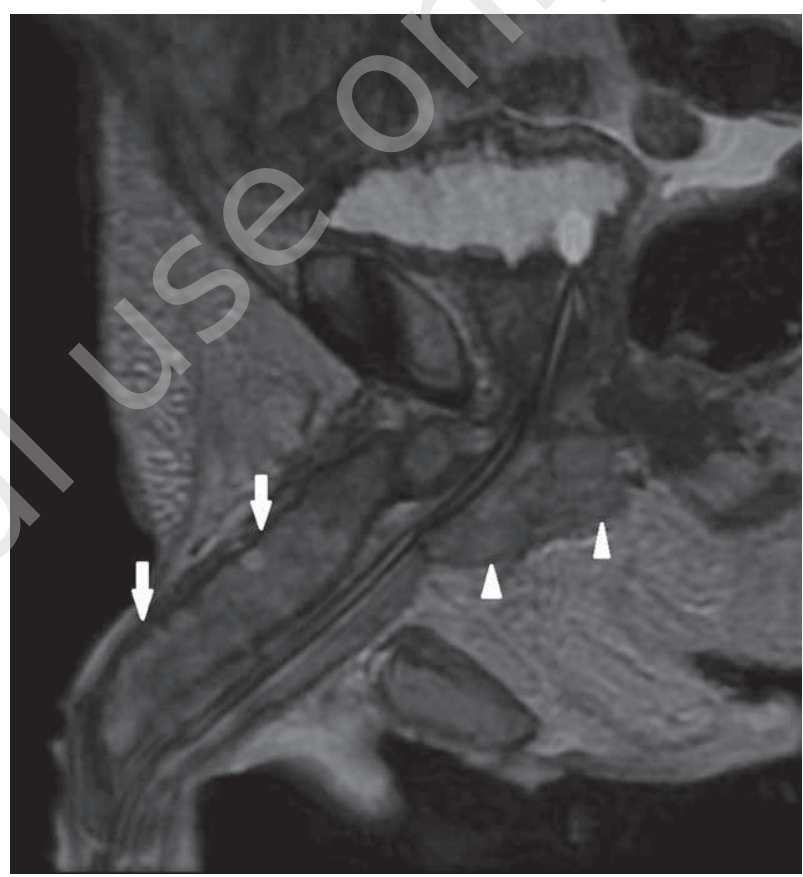

Conservative treatment and antibiotic therapy administered with 2 units red cell concentrate transfusion improved the general health condition of the patient, alleviated localised symptoms and had positive impact on clinical and laboratory parameters.

The patient did not give his consent for further diagnostic procedures (colonoscopy) and was discharged home on his own request.

During the 6 weeks follow-up, the patient retained the Foley catheter, however he did not continue the prescribed course of treatment. The patient reported an improvement in his general condition and a slight reduction of the swelling of the penis.

\section{DISCUSSION}

Deep dorsal penile vein thrombosis is hardly ever described and there are no firm recommendations on the diagnosis and treatment of the condition [1]. Only few cases of PDDVT have been described [1, 3, 5]. Literature describes slightly more cases of corpora cavernosa thrombosis that may present with the 
deep dorsal penile vein thrombosis or on their own $[2,6-8]$. UItrasound examination followed by magnetic resonance imaging can detect corpora cavernosa thrombosis, however changes in the deep dorsal penile vein may go undetected $[6,9]$. In the case discussed, colour Doppler examination showed a minimal blood flow in corpora cavernosa and no blood flow in the deep dorsal penile vein. Magnetic resonance imaging detected thrombosis and a $3 \mathrm{~cm}$ long tumour metastasis in the crus of the penis. The results of the clinical examination together with the drawn conclusions facilitated the formulation of a correct final diagnosis. The course of the treatment was in line with recommendations made by other authors $[1-3,5,8]$.

As suggested by Calanci et al., the patient was given unfractionated heparin instead of low molecular weight heparin [5]. It was decided not to follow the recommendations of Albrecht and Stackl [10], who suggest injections of ethylephrine hydrochloride, due to months' long medical history and the patient's age.

Due to the fact that venous thromboembolism (VTE) is a serious problem in oncology as it is the most common complication and the second cause of death in patients suffering from malignant cancer [11], a thorough examination of the chest and the veins in lower limbs was conducted, however, no changes pointing to VTE were found.

It should be stressed that the patient described in this particular case, initially presented with first symptoms of penile cavernous thrombosis. An in depth diagnostic process facilitated the detection of advanced metastatic cancer of the colon, which turned out to be the primary disease, and the thrombosis of the veins and corpora cavernosa of the penis should be diagnosed as the secondary metastatic lesions detected by MRI and biopsy.

The lack of consent of the patient to undergo colonoscopy was not a unique case, as about $15 \%$ of the patients suffering from colorectal cancer refuse oncological treatment [12].

Literature does not describe correlation between deep dorsal penile vein thrombosis and colorectal cancer. Weyne et al. pointed that deep dorsal penile vein thrombosis and the corpora cavernosa thrombosis are usually idiopathic [7]. Only few authors mentioned haematological factors $[3,13]$. In one of the publications corpora cavernosa thrombosis was a consequence of prostate abscess [5]. According to available sources, the case described in this paper is the first one when deep dorsal penile vein thrombosis and the cavernous vein thrombosis were the consequence of the spread of the colorectal cancer.

\section{SUMMARY}

Deep dorsal penile vein thrombosis is a rare disease of the penis and is most often a symptom of a coexisting prevalent disease, such as cancer or haematological diseases. Conservative treatment brings only slight improvement and it is necessary to expand diagnostic process in order to detect cancer.

\section{References}

1. Sarker B. A rare case report of spontaneous thrombosis of the deep dorsal vein of penis. The British Medical Ultrasound Society. Radiology BMI Woodlands Hospital. Poster Presentations January 2018.

2. Horger DC, Wingo MS, Keane TE. Partial segmental thrombosis of corpus cavernosus: case report and review of world literature. Urology. 2005; 66: 194.

3. Schmidt BA, Schwartz T, Schellong S. Spontaneous thrombosis of the deep dorsal vein in a patient with thrombophilia. J Urol. 2000; 164: 1649.

4. Al-Mwalad M, Loertzer H, Wicht A et al. Subcutaneous penile vein thrombosis (penile Mondor's disease) - pathogenesis, diagnosis et therapy. Urology. 2006; 67: 586-8.

5. Calanca L, Alatri A, Schaller MD et al. Deep vein thrombosis of the penis: an unusual but severe complication of prostatic abscess. VASA. Zeitschrift fur Gefasskrankheiten. 2013; 42(3): 214-7. https://doi.org/10.1024/0301-1526/a000269.

6. Thiel R, Kahn T, Vogeli TA. Idiopathic partial thrombosis of the corpus cavernosum. Urol Int. 1998; 60: 178-80.

7. Weyne E, Schillebeeckx C, Jamaer C et al. Idiopathic partial thrombosis (IPT) of the corpus cavernosum; a hypothesis-generating case series and review of the literature. J Sex Med. 2015; 12: 2118-25.

8. Głuchowski J, Bławat A, Kordasz J et al. Partial segmental thrombosis of the corpus cavernosum. Cent European J Urol. 2011; 64(4): 264-5. https:// doi.org/10.5173/ceju.2011.04.art19.

9. Goeman L, Joniau S, Oyen R et al. Idiopathic partial thrombosis of the corpus cavernosum: conservative management is effective and possible. Eur Urol. 2003; 44(1): 119-23. https://doi.org/10.1016/s0302-2838(03)00217-3.

10. Albrecht W, Stackl W. Treatment of partial priapism with an intracavernous injection of etilefrine. JAMA. 1997; 277(5): 378. 
11. Wojtukiewicz MZ, Sierko E, Tomkowski W et al. Guidelines for the prevention and treatment of venous thromboembolism in patients with cancers treated conservatively. Oncol Clin Pract. 2016; 12: 67-91.

12. Copija A, Głogowski Ł, Walkiewicz K et al. Variability of clinical characteristic of colorectal cancer patients - analysis of selected parameters. OncoReview 2018; 3(31): 80-5. https://doi.org/10.24292/01.0R.290818.

13. Shen H-L, Liu S-P, Wang S-M et al. Elevated plasma factor VIII coagulant activity presenting with thrombophlebitis of the deep dorsal vein of the penis. Int J Urol. 2007; 14: 663-4. https://doi.org/10.1111/j.1442-2042.2007.01789.x.

Authors' contributions: All authors have equal contribution to the paper and research behind.

Conflict of interests:

Authors declare no conflict of interest.

Financial support:

There was no financial support.

Ethics:

The authors had full access to the data and take full responsibility for its integrity. 